\title{
Future Skills für Future Organisations: Analyse zukünftiger Organisationsmodelle
}

Die im vorhergehenden Kapitel ausgeführte „Drift to Self-Organisation“ die sich in allen Lebensbereichen zeigt, und sich in neuen Lebens-, Lern- und Arbeitsmodellen ausdrückt, führt zu neuen Anforderungen an Individuen in der Gesellschaft insgesamt und in Organisationen. In diesem Kapitel zeigen wir Beispiele und Ansätze aus dem Bereich des Selbstmanagements und der Organisationstheorie, in denen Selbstorganisation zugrunde liegt und Future Skills eine besondere Rolle spielen.

Betrachtet man Organisationen und analysiert, inwieweit sie auf Selbstorganisation ausgerichtet sind, fällt zunächst einmal ins Auge, dass zwischen den Polen „Verlässlichkeit“ und „Anpassungsfähigkeit“ vielfach der Glaube vorherrscht, Verlässlichkeit stärker betonen und ausprägen zu müssen als Anpassungsfähigkeit. In der Future Skills Studie zeigt sich aber, dass diese Denkweise gerade in Future Organisations immer stärker in Frage gestellt wird. Andererseits gibt es immer mehr empirische Erkenntnisse, die zeigen, wie wichtig die psychologische Komponente der Identifikation mit dem was Mitarbeiterinnen und Mitarbeiter tun, auch für die Arbeitszufriedenheit und für die Produktivität ist.

Die deutsche Unternehmensberatung Gallup Deutschland, ein forschungsbasiertes Beratungsunternehmen und Spezialist für die Schnittstelle Ökonomie und Psychologie hält seine jährlichen Ergebnisse im sog. „Engagement Index“ fest. Für 2016 konstatiert die Studie, dass die deutschen Arbeitnehmerinnen und Arbeitnehmer zufrieden sind mit ihrem Leben und die ökonomische Lage als positiv bewerten, kaum um ihren Arbeitsplatz fürchten und dass auch die Arbeitseinstellung positiv ist (Nink 2014). Siebenundsiebzig (77) Prozent würden selbst dann weiterarbeiten, wenn sie nicht auf das Geld angewiesen wären (Nink 2014). Das sind sieben Prozent punkte mehr als noch 2010. Dennoch ist die Mehrheit der Mitarbeiterinnen und Mitarbeiter emotional kaum an ihren Arbeitgeber gebunden. Das wirkt sich direkt auf wichtige Wettbewerbsfaktoren wie Fehlzeiten, Produktivität, Rentabilität, Qualität und Kundenbindung aus. Denn Arbeitnehmerinnen und Arbeitnehmer die sich emotional nicht an ihren Arbeitgeber gebunden fühlen, zeigen weniger 
Eigeninitiative, Leistungsbereitschaft und Verantwortungsbewusstsein und sie schweigen zudem häufiger zur Fehlentwicklung. Laut aktuellem „Engagement Index" hat jede/r dritte Mitarbeiterin und Mitarbeiter in den letzten 12 Monaten gegenüber seinem Vorgesetzten/ ihrer Vorgesetzten mindestens einmal schwere Bedenken nicht geäußert; bei den Mitarbeiterinnen und Mitarbeitern ohne emotionale Bindung schwieg sogar fast jeder Zweite. Diese Themen, wie emotionale Bindung, das Empfinden von ungerechtfertigten Hierarchien, die im Falle von komplexen Problemsituationen fachlich nicht ausreichend fundierte Entscheidungen treffen, werden von Organisationen und Unternehmen aller Größen derzeit intensiv diskutiert.

Auch die NextSkills Studie zeigt, dass das Thema emotionaler Bindung von Mitarbeiterinnen und Mitarbeiter an ihre jeweilige Organisation eines der wichtigsten Führungsthemen ist, welches auch über die Motivation der Mitarbeiterinnen und Mitarbeiter bestimmt, sich einzubringen. Dabei spielen in den Daten der Interviews aller Befragten immer wieder zwei Entwicklungsbereiche eine wesentliche Rolle: Wertemanagement und neue Führungskonzepte. Dabei bezieht sich das Wertemanagement auf die Wertschätzung von Diversität und unterschiedlichen Begabungen, Fähigkeiten und Kompetenzen, sowie Interessen, um „shared cognition $^{\text {"32 }}$ in Teams herzustellen und Teamleistungen zu erhöhen. Zusätzlich geht es um Identifikation, Motivation, den Culture-Fit und das Transportieren der für die Organisation jeweils zentralen wichtigen Werte. Diese nehmen die Funktion eines „sozialen Kits" ein, der durch die reine Organisationszugehörigkeit nur noch in schwindendem Maße gegeben ist, da die Verbindlichkeit und zeitliche Dauer der Organisationangehörigkeit immer wieder in Frage gestellt und in Normalbiographien immer schneller episodisch verhandelt wird. Führungskonzepte für Future Organisations beschäftigen sich vor allem mit Kommunikation, Feedback, Hierarchieabbau und dezentraler, individueller Verantwortungsübernahme. Die Future Skills Studie zeigt, dass Instrumente wie Coaching, Mentoring, auch das Initiieren von kollegialen Kommunikationsnetzwerken und das Moderieren von self-supporting Structures in Organisationen immer wichtiger werden. Führungskräfte werden dabei vor neue, bislang nicht so stark im Vordergrund stehende Herausforderungen gestellt. Neue Qualifizierungserfordernisse treten dabei auf. Mindful Leadership, systemische Beratungs- und Coachingansätze, Gewaltfreie Kommunikation und kommunikative Moderation sowie kollegiale Beratung nehmen an Bedeutung zu, gegenüber hierarchischem Delegieren und „Assign-Control Ansätzen“. Zwei Fallbeispiele illustrieren diese Instrumente und Organisationsformen.

32 Das Konzept des „shared cognition“ bezieht sich auf das Konzept des situierten Lernens und auf Peer-Learning (Brown, Collins \& Duguid 1989; Lave \& Wenger 1991). 


\section{Interesting Practice: Daimler}

Wie sehr das Thema Hierarchie und alternative Ansätze aktuell bekannte Großunternehmen beschäftigt, zeigt beispielsweise auch der Beitrag von Daimler-Benz Chef Zetsche. Eine Startup Kultur soll neuen Spirit ins Unternehmen bringen. Mit diesem Ansatz will man mehr Basisdemokratie wagen. Im Programm Leadership 2020 geht es um neue Führungskultur. Die Impulse kommen von 150 Mitarbeiterinnen und Mitarbeitern aus 24 Nationen, aller Bereiche und Rangstufen, von Sachbearbeiterinnen und Sachbearbeitern über Meisterinnen und Meister bis hin zu Direktorinnen und Direktoren. In acht Teams werden Ideen und Visionen entwickelt, wie zukünftig Führung bei Daimler aussehen kann. Hinterfragt werden die Hierarchiestruktur, die Meetingkultur, die Leistungsbewertung und es gibt nur eine einzige Vorgabe - es gibt keine Vorgabe.

\section{Good Practice: Spotify (Open Access für Musik) ${ }^{33}$}

Ein Beispiel für Selbstorganisation in Organisationen ist der Musik Streaming Dienst Spotify. Bei Spotify sind agile Unternehmensstrukturen Programm. Gute Mitarbeiterinnen und Mitarbeiter, so wird es bei Spotify gesehen, treffen in 70 Prozent aller Fälle dieselben Entscheidungen wie ihr/sein Chef. In 20 Prozent fällt sie/er bessere Entscheidungen, weil sie/er von der Sache mehr Ahnung hat. Nur in lediglich 10 Prozent liegt sie/er daneben. Diese Managementprinzipien wurden von Daniel Ek geprägt. Er ist Gründer und Vorstandsvorsitzender von Spotify.

Die Spotify Story in Kurzfassung: Daniel Eks Stiefvater, ein Elektroingenieur, führte den Jungen früh in die Welt der Computer ein. Schon als Grundschüler schrieb er auf einem Commodore C64 erste Programme, gründete mit 14 aus dem Kinderzimmer heraus seine erste Firma und kreierte Unternehmenswebsites cooler als die kommerziellen Webagenturen in der schwedischen Hauptstadt. Die Firma wuchs. Mit 19 verkaufte Ek den Webdienstleister. Ramge (2015) berichtet, dass er ein Informatikstudium begann, es aber schnell wieder abbrach und schließlich Chef der Softwarefirma uTorrent wurde, mit deren Programmen weltweit Musik und Filmdateien illegal getauscht werden konnten. In dieser Zeit kam er auf die Idee für Spotify. Er fand Investoren und 12 Mio. Euro Risikokapital; keine andere Musik Streaming Plattform wuchs so schnell wie Spotify. Ramge (2015) analysiert, dass das auch viel mit Eks speziellem Führungsmodell zu tun hat, durch den guten Programmierer zu Spotify kommen und dort bleiben. Nur sie sind in der Lage, den Komfort zu schaffen, für den Internetzuhörerinnen und -zuhörer im Zeitalter der

33 Darstellung des Fallbeispiels aus der Zeitschrift Brandeins in Anlehnung an Ramge (2015). 
Kostenloskultur zu zahlen bereit sind. Wer die Besten dieser Zunft anziehen will, muss ihnen viel Freiraum geben, das wusste Ek. Er gehörte ja selber dazu. Freiraum $\mathrm{zu}$ geben war für ihr kein Problem. Er ist selten längere Zeit an einem Ort, was bei einem globalen Unternehmen mit zwei Hauptsitzen und fünf Entwicklungsstandorten verständlich ist.

Ramge (ebenda) führt aus, dass es bei Spotify 60 sogenannte agile Coaches gibt. Moderatorinnen und Moderatoren unterstützen die Teams ohne interne Hierarchien dabei, die richtigen Entscheidungen zu treffen und die Arbeit so zu organisieren, dass jedes Team produktiv und jedes Teammitglied glücklich ist. So kommen bei Spotify 1200 technische Entwicklerinnen und Entwickler in Stockholm, Göteborg, New York, Boston und San Francisco ohne Chef aus. Bei Spotify verdoppelt sich die Mitarbeiterzahl alle 12 Monate. Die Herausforderung besteht darin, die Kultur mit viel Entscheidungsfreiheit und Teamgeist in kleinen Einheiten zu erhalten, ohne dass das Produkt und der Laden auseinanderfallen. Spricht man mit Spotifylern, so kommt man zu dem Schluss, die Unschärfe sei Teil des Systems (Ramge 2015).

\section{B 4.1 Selbstorganisation als Managementprinzip}

Spotify ist nach agilen, holokratischen Prinzipien organisiert, die wir in Abbildung 22 dargestellt haben. Mit dem Thema Führung verhält es sich dabei so, wie mit der Programmiermethode SCRUM ${ }^{34}$ und bei deren Ansatz zur Verbesserung von Softwareprogrammen: Man definiert ein Ziel, stellt aber keinen Plan auf, sondern tastet sich heran. Per try and error werden Ideen ausprobiert. Funktioniert eine, wird sie weiterverfolgt; zündet sie nicht, wird sie fallen gelassen. Ein weiteres wichtiges Prinzip besteht in der no blame culture, also darin, ohne Schuldzuweisungen $\mathrm{zu}$ arbeiten.

Teams heißen nicht Teams, sondern Squads. Eine dieser Einheiten hat zwischen sechs und 20 Mitarbeiterinnen und Mitarbeiter (siehe Abbildung 22: Agile, holokratische Strukturen in self-managed Organisations). Sie ist immer interdisziplinär besetzt mit klassischen Entwicklerinnen und Entwicklern, Expertinnen und Experten für User Experience und Tests sowie Designerinnen und Designern. Sie hat keinen Chef aber einen sogenannten Product-owner. Sie/Er gibt Themen vor und organisiert die vielen gemeinsamen Konferenzen und die zum Teil sehr

34 Scrum (englisch für Gedränge) ist ein Vorgehensmodell des Projekt- und Produktmanagements, insbesondere zur agilen Softwareentwicklung. Es wurde ursprünglich in der Softwaretechnik entwickelt, ist aber davon unabhängig. 

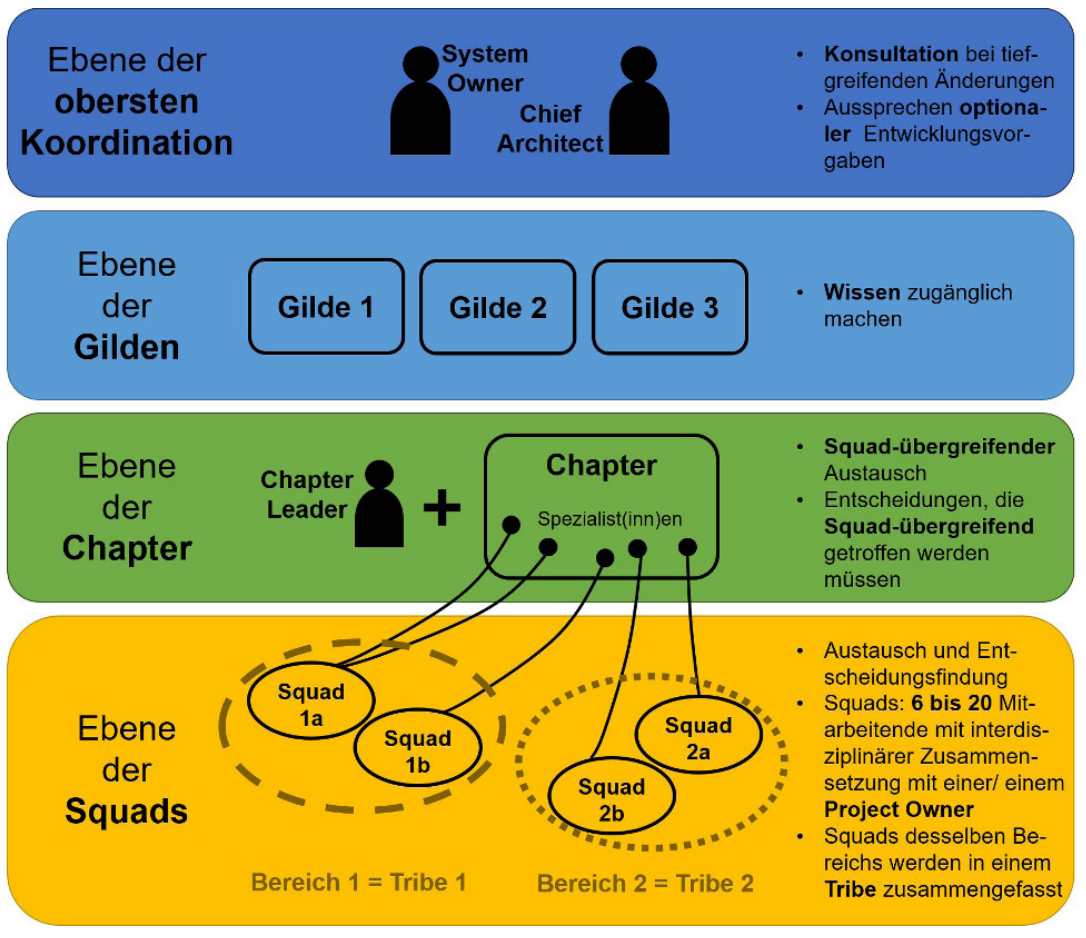

Abb. 22 Agile, holokratische Strukturen in self-managed Organisations

emotionalen Sitzungen an Freitagnachmittagen, an denen die Woche bilanziert wird. Ein agiler Coach achtet darauf, dass die Regeln eingehalten werden. Jedes Mitglied kann Entscheidungen herbeiführen, es muss dafür nur die Kolleginnen und Kollegen von ihrer/seiner Idee überzeugen. Squads die im gleichen Bereich arbeiten, gehören demselben "Tribe“ an. So ein Stamm darf nicht mehr als 150 Angehörige haben, damit er nicht zu unübersichtlich wird (siehe Abbildung 22).

Ramge (2015) beschreibt, dass die Mitglieder eines Tribes regelmäßig zusammen kommen, um Informationen auszutauschen und Entscheidungen zu treffen, die alle angehen. Die Spezialistinnen und Spezialisten besprechen zudem Themen bei denen aus technischen Gründen Konsens hergestellt werden muss. Diese Spezialistinnen und Spezialisten gehören zusätzlich einem squadübergreifenden Chapter an, das einen "Chapter Leader" hat. Doch die/der ist nur in formellen Fragen wie etwa bei Urlaubsanträgen mit Autorität versehen, sonst hat sie/er nur eine beratende 
Funktion inne. Die Ebene über den Stämmen wird von "Gilden“ eingenommen. Sie haben die Aufgabe, Wissen überall im Unternehmen zugänglich zu machen. Die oberste Koordination fällt zwei Personen zu: einer/ einem sogenannten system owner und einer/ einem chief architect. Größere Änderungen im System müssen die Squads mit diesen beiden abstimmen. Feste Regeln gibt es dabei aber nicht. Manchmal machen die obersten Koordinatorinnen und Koordinatoren Entwicklungsvorgaben, manchmal setzen die selbstbewussten Squads ihre Vorstellungen durch. Oder aber der Gründer oder der Chef-Designer Peter bricht alle Regeln, bzw. spricht ein Machtwort (Ramge 2015).

\section{Interesting Practice Deutsche Telekom}

Im AI Blog der Deutschen Telekom (Bäumler 2017) berichtet Michael Kaselow, agiler Coach bei der Deutschen Telekom, von der Erfahrung mit Holokratie im Unternehmen: „Wir haben das Spotify-Modell für uns adaptiert. Die Herausforderung dabei ist, dass die Struktur nicht wie bei Spotify organisch gewachsen ist. Sie wurde vielmehr aufgesetzt und wir als agile Coaches müssen dafür sorgen, dass das funktioniert. Da es relativ wenig Material über diese Art und Weise der Organisation gibt, pflegen wir immer noch eine Atmosphäre des Learning by doing und passen alles auf unsere Bedürfnisse an. Wenn wir an neuen Themen oder Produkten arbeiten, müssen wir Squads oder Tribes oder auch die Chapter neu aufsetzen." Im Projekt eLIZA sind es aktuell etwa 15 Squads, die auf vier Tribes verteilt sind. ${ }^{35}$ Zudem gibt es noch so genannte Chapters, die sich aus den Leuten rekrutieren, die in den Squads und Tribes arbeiten und welche die gleiche Profession haben. Das können zum Beispiel Entwicklerinnen und Entwickler, User Experience-Expertinnen und Expertenen, Designerinnen und Designer oder Testerinnen und Tester sein. Sie tauschen sich über die Squad-Grenzen hinaus aus und entwickeln gemeinsame Methoden.

Kaselow sagt: „Es muss ja nicht jeder Entwicklungs-Squad eine eigene Testumgebung aufsetzen. Vieles kann man sharen oder gemeinsam aufbauen - dafür sind die Chapters verantwortlich." Regelmäßig trifft man sich zum Campus, einer Veranstaltung, bei der die einzelnen Squads Meilensteine vorstellen und gelegentlich sogar externe Expertinnen und Experten zu bestimmten Themen sprechen. Neben der internen Weiterbildung steht der informelle Austausch im Vordergrund - im

35 eLIZA ist der der Name eines Innovations-Projekts der Deutschen Telekom mit der Aufgabe, eine Künstliche Intelligenz (KI oder englisch AI für Artificial Intelligence) zu entwickeln. Der Name eLIZA wurde übernommen von einem 1966 von Joseph Weizenbaum entwickelten Computerprogramm. Es sollte die Möglichkeiten der Kommunikation zwischen einem Menschen und einem Computer über natürliche Sprache aufzeigen. 
Bewusstsein, dass die unterschiedlichen Team-Einheiten, also Squads, Tribes und Chapters den Herausforderungen nur gemeinsam begegnen können (ebenda).

Die Managementkonzepte, die auf Selbstorganisation basieren sind fundamentale Experimentierfelder für Organisationen. Die Future Skills Studie 2018 zeigt, dass Selbstorganisation als Managementprinzip bereits stark verbreitet ist, jedoch ohne, dass explizit weitergehende Organisationsformen, wie beispielsweise Holokratie dafür eingeführt werden.

Dabei ist festzuhalten, dass diese bei Startups und kleinen Unternehmen oftmals natürlich eingeführt werden, während es zur Transformation von größeren und traditionellen Organisationen bisher nur wenig oder keine Erkenntnis über Erfolgsfaktoren gibt. Soziokratie, Demokratie und Holokratie sind aktuell in aller Munde. Die drei Konzepte können gemäß Frederic Laloux als die nächste Form der Evolution von Unternehmen angesehen werden, wie er in seinem Buch Reinventing Organizations: Ein Leitfaden zur Gestaltung sinnstiftender Formen der Zusammenarbeit (2015) darstellt. Demnach scheint die Holokratie die perfekte Antwort auf eine immer schnelllebigere und komplexere Arbeitswelt zu sein. Sie ermöglicht es Unternehmen, flexibel auf Veränderungen im Außen (oder Innen) zu reagieren und steigert zugleich die Innovationskraft der Organisation. Die Mitarbeiterinnen und Mitarbeiter sind eigenständiger, genießen persönliche Freiheit sowie einen hohen Grad an Eigenverantwortung. Dadurch sind sie langfristig zufriedener, motivierter, gesünder und produktiver. Freiheit und Selbstverantwortung - das sind wichtige Stichworte für aktuelle Employer Branding Konzeptionen.

Jedoch ist nicht klar ob und wenn ja welche zukünftige Organisationform sich durchsetzen wird - gerade in großen, traditionell strukturierten Organisationen. Im Folgenden werden Vor- und Nachteile der drei Modelle erläutert.

\section{B 4.1.1 Soziokratie in Organisationen}

Soziokratie geht bei der Steuerung von Entscheidungen im Unternehmen davon aus, dass alle Beteiligten sehr gleich sind. Eine Entscheidung ist getroffen, sofern kein ernstes Gegenargument mehr vorliegt. Die Methode fordert also Eigenmotivation und ein kooperatives Miteinander sowie Selbstverantwortung. Sie zielt außerdem nach dem Managementprinzip Y (McGregor 1960) darauf ab, dass sich Mitarbeiterinnen und Mitarbeiter wohlfühlen und deswegen im Unternehmen nach Selbstverwirklichung streben. Managementtheorie X (ebenda) bedeutet hingegen, dass der Mensch grundlegend eine Abneigung gegen Arbeit angestrebt hat und eine Führungskraft ihn zur Arbeit zwingen muss. Im Vergleich zu Managementtheorie X, besagt Managementtheorie Y, dass Arbeit einen hohen Stel- 
lenwert bei Mitarbeiterinnen und Mitarbeitern hat und eine wichtige Quelle von Selbstzufriedenheit darstellt.

\section{B 4.1.2 Holokratie - Agilität und Verantwortung}

Das Konzept von Robertson (2015) ist aktuell in aller Munde. Es regelt die Führung von Organisationen durch eine Transparenz, die es jeder und jedem durch alle Ebenen und Prozesse hindurch ermöglicht, sich einzubringen. Es setzt den Zweck der Organisation in den Mittelpunkt und nicht den Profit. Robertson zeigt eindrucksvoll, wie Managerinnen und Manager in einer Holokratie nicht die Position und den Status einer Managerin, bzw. eines Managers einnehmen, sondern dessen/deren Rolle und Verantwortung. Im Zentrum der Holokratie steht ein Leitungskreis, der alle Aktivitäten und Probleme steuert. Jede und jeder, die/ der sich ins Unternehmen einbringen möchte, darf an diesem teilnehmen und nimmt dort eine bestimmte Rolle ein. Um den Leitungskreis sind diverse weitere Rollen aufgebaut, z. B. die/der Business Developer oder die/der Consultant. Diese Rollen können aus einer oder mehreren Personen bestehen und ständig wechseln. Sollte nun eine Anfrage von außen, also von der Kundin oder dem Kunden kommen, so reagiert der jeweilige Kreis entsprechend auf die Anfrage und entscheidet darüber autonom und selbstständig. Die Kundin/ der Kunde möchte z. B. einen neuen Auftrag vergeben und die betreffende Person, die mit der Anfrage zu tun hat, wechselt von der Rolle Consultant in die Rolle Vertrieb. Der Leitungskreis darf nun, wenn gewünscht, diese Person unterstützen, beispielsweise bei der Suche nach einer/ einem geeigneten Mitarbeitenden. Sobald diese Situation geklärt ist, nimmt die Person wieder die Rolle der/des IT Consultants ein. Jedoch hat sich nun daraus ein neuer Kreis gebildet, das aus zwei Personen, Person X und der/ dem neuen Consultant für den Auftrag besteht und klar der Kundin/ dem Kunden zugeordnet ist. Wir haben also verschiedene Kreise im Unternehmen, die sich mit einem bestimmten Thema befassen. Auch die gesamte Organisation ist als Kreis zu verstehen. Abseits der Kreise gibt es viele verschiedene Rollen. Als Mitarbeiterin und Mitarbeiter kann ich in mehreren Kreisen verortet sein und stets etwas zum Zweck der Organisation beitragen. So wird das Unternehmen dynamisch gesteuert und orientiert sich vorrangig am Zweck der Organisation. Rollen ersetzen Positionen und Hierarchie. Zudem steht eine lebendige Struktur über starren Organigrammen.

Die Holokratie besteht in einer Analogie zur Biologie aus verschieden Kreisen, sogenannten Holons, die anderen Dinge umschließen. So befinden sich in einem Holon mehrere Moleküle (Rollen) und ein Molekül besitzt wiederum mehrere Atome (ebenda). Die Atome und Moleküle in einem Holon ändern sich zwar nicht, können 
aber durch einen neuen Verbund neue Eigenschaften erschließen. Das klappt in der Natur seit Millionen von Jahren. Agilität und Holokratie haben viel gemeinsam.

\section{B 4.1.3 Das demokratische Unternehmen}

Der Ansatz der Demokratie in Unternehmen vertritt als erstes die Fragen „Wer führt mich?“, „Wer vertritt mich?“ und „Wie bin ich am Unternehmen beteiligt?“. Hier wird ebenfalls mit dem Faktor Zeit bei der Führung experimentiert. Das zweite Thema ist die Selbstbestimmtheit: „Wo arbeite ich, wann und mit wem?“ (Sattelberger 2015). Es geht also um mehr Mitspracherecht für die Mitarbeiterinnen und Mitarbeiter sowie um Chancenfairness. Nach den Autoren des Buches „Demokratische Unternehmen“, Sattelberger et al. (2015) geht es darum, dem Wunsch von Mitarbeiterinnen und Mitarbeitern an der Strategieentwicklung ihres Unternehmens mitwirken zu dürfen, nachzukommen und sie über ihre eigene Arbeitssituation entscheiden zu lassen. Es stellt also die Gruppenentscheidung in den Vordergrund. Das Ziel ist nicht eine Mehrheitsentscheidung zu erwirken, so der Autor, sondern die Position der Gruppenmitglieder zu verändern, sodass sich ihre Stimmen zur kritischen Masse einer Option vereinen. Viele Unternehmen überlegen, wie eine solche Demokratie aussehen könnte. Eine aufgegebene These in verschiedenen Quellen lautet, digitale Technologien erleichterten die Mitbestimmung. Kann so die Zukunft aussehen? Mitarbeiterinnen und Mitarbeiter wählen Managerinnen und Manager, stimmen über neue Produkte ab, entscheiden über Arbeitszeiten sowie über Kundinnen und Kunden. Aktuell ist dieses Thema noch stark umstritten und bietet Raum für weitere Forschung. Es zeigt sich aber, dass viele Mitarbeiterinnen und Mitarbeiter wenig Interesse auf Fremdbestimmung haben. Durch digitale Technologien sind Abstimmungsprozesse einfacher geworden. Der CEO von Microsoft Deutschland sagte dazu: „Früher suchten wir Mitarbeiterinnen und Mitarbeiter, die tun was wir sagen, heute suchen wir Mitarbeiterinnen und Mitarbeiter die machen, was wir nicht sagen“ (ebenda).

\section{B 4.2 Self-Management und Agilität in der Praxis? Zum Stand der Forschung}

In ihrem Beitrag zur Holokratie im Harvard Business Review spannen Bernstein et al. (2016) einen Orientierungsrahmen auf, in dem sie ein Spannungsfeld von Stabilität und Zuverlässigkeit auf der einen Seite und Anpassungsfähigkeit auf 
der anderen Seite aufzeigen. Sie argumentieren, dass holokratische Organisationsformen kein Allheilmittel seien und dass deren Umsetzung davon abhängig gemacht werden sollte, wie sich die Rahmenbedingungen in Unternehmen bzw. Teilorganisationen gestalten:

- Sind die Anforderungen an Stabilität und Zuverlässigkeit hoch, braucht es große langfristig wirksame Investitionen. Braucht es beispielsweise in einem Maschinenpark eine Gesamtsteuerung über eine langfristige strategische Planung, dann sind holokratische Organisationsformen nicht unbedingt zielführend.

- Bewegt sich das Unternehmen/ die Teilorganisation in einer unsicheren Umwelt mit wechselnden Anforderungen? Ist das Produkt-Serviceportfolio breit und diversifiziert? Ist eine Gesamtsteuerung auf der Basis weniger Leitlinien möglich? Dann können holokratische Organisationsforen durchaus sinnvoll sein. Aber auch in diesem Fall gibt es durchaus noch offene Fragen: Wie wird eine Gesamtkoordination der einzelnen Teileinheiten/ Kreise sichergestellt? Wer übernimmt die Gesamtverantwortung nach außen? Welche Vergütungsmodelle passen zu so einer veränderten Organisation und den neuen Mechanismen der Aufgabenverteilung?

Insgesamt zeigt das Agilitätsbarometer einer Studie von Haufe und Promerit (Anderson et al. 2017), dass Agilität in deutschen Unternehmen sich noch nicht als dominantes Managementprinzip durchgesetzt hat. 90 Prozent der Mitarbeiterinnen und Mitarbeiter und 70 Prozent der Führungskräfte geben an, nie agile Methoden zu nutzen. Dabei zeigen sich kaum Veränderungen gegenüber den Befragungsergebnissen aus dem Vorjahr. Scrum swarming oder Holokratie kennen 80 Prozent der Mitarbeiterinnen und Mitarbeiter nicht. Etwas besser sieht es im Hinblick auf Design Thinking (57\%) und fluiden Strukturen (61\%) aus. Anhand des Fallbeispiels von Spotify haben wir schon Charakteristika von holokratisch organisierten Organisationen kennengelernt.

Was ist weiterhin charakteristisch für Self-Managed Organizations (SMO)? Self-Managed Organizations arbeiten mit Self-Managed Teams: dabei sind die Verantwortlichkeiten für die Arbeit zwischen den Mitgliedern der Teams aufgeteilt. Die Mitglieder teilen sich die Verantwortung mit Blick auf die Art und Weise der Zielerreichung, Ressourcennutzung und ein Ownership an Information und Wissen, die sich auf die Arbeitsaufgaben beziehen. Bei den Organisationen und Unternehmen, die den Sprung in die Agilität und in die Selbstorganisation gewagt haben, können Variationen des Selbstmanagements erkannt werden. Darunter fallen Unternehmen wie beispielsweise Morning Star, ein Hersteller von Tomatenprodukten, Valve, ein Entwickler von Videospielen und Spieleplattformen, $W$. 
L. Gore, ein stark diversifizierter Hersteller und der schon erwähnte Zappos. Die Variationen der unterschiedlichen Selbstorganisationsgrade und -ausformungen sind Ausdruck der jeweils spezifischen Management- und Organisationskontexte. Das bekannteste und am besten spezifizierten System für Self-Managed Organizations und Self-Managed Teams ist das bereits beschriebene System der Holokratie (siehe Kapitel B 4.1.2 Holokratie - Agilität und Verantwortung). Self-Managed Models (SOM), haben typischerweise drei Charakteristika:

1. Teams sind die Struktur: In der Holokratie werden sie „Circles“ genannt. In der "Podularity" wird von "Pods" gesprochen, bei Valve von "Cabals" und in vielen anderen Unternehmen einfach von Teams. Aber wie auch immer sie heißen, Teams sind die Basiskomponenten für die Gesamtorganisation - nicht Individuen, nicht Abteilungen, Departments oder Divisions. Die Rollen werden in den Teams kollektiv entwickelt, definiert und den einzelnen Arbeitsaufgaben zugeordnet. Wie auch in traditionellen Organisationsformen, gibt es auch in Self-Managed Organizations unterschiedliche Teams für unterschiedliche Projekte, Funktionen (beispielsweise für Finanzen, technische Entwicklung, Vertrieb oder unterschiedliche Segmente (Kunden, Produkte, Services)). Bei Zappos wurden die 150 Departmenteinheiten nach diesem Vorbild in etwa 500 Circles umgewandelt. Die so entstehende Modularität ist sehr viel flexibler als in Linien hierarchisch aufgebaute Organisationen. Dabei können Teams ad hoc neu eingerichtet oder wieder aufgelöst werden, entsprechend der aktuellen Organisationsbedürfnisse.

2. Teams entwickeln und leiten sich selbst: Obwohl Self-Managed Organizations traditionelle Hierarchiestrukturen meiden, sind Teams trotzdem in größere Strukturen eingebettet, die sie mitbestimmen können. Holokratische Organisationen verabschieden dazu eine Konstitution, eine Organisationscharta, eine Konstitution die in der Regel ein „living document“ darstellt, in dem die Regeln darüber, wie Circles eingerichtet, entwickelt, verändert und aufgelöst werden, niedergeschrieben sind. Somit managen die Circles nicht nur sich selbst, sondern es gibt übergreifende Regeln, wie sie „designed“ und geleitet werden. Die Konstitutionen und Chartas bestimmen allerdings nicht wie Mitarbeiterinnen und Mitarbeiter ihre Aufgaben zu erfüllen haben. Sie geben lediglich einen Rahmen dafür vor, wie Circles entstehen, geformt werden und miteinander arbeiten, wie sie Rollen identifizieren und zuordnen, welche Grenzen diese Rollen haben und wie sie untereinander interagieren können. Bei Morning Star schreiben die Mitarbeiterinnen und Mitarbeiter in Teams sogenannte CLOUS (collegue letters of understanding). In diesen werden die Verantwortlichkeiten, Aktivitäten und Ziele festgehalten, die in den Teams angestrebt werden und auch 
Kriterien und Messverfahren zur Evaluierung der Leistungsmessung. Clous sind also Vereinbarungen der Circles miteinander.

3. Führung ist hochkontextualisiert: In Self-Managed Organizations ist die Führung zwischen unterschiedlichen Rollen aufgeteilt, nicht zwischen Individuen. Dabei haben Akteurinnen und Akteure normalerweise viele Multiplayer-Rollen in verschiedenen Teams inne. Wenn Arbeitszusammenhänge sich ändern, ändern sich auch Führungsverantwortlichkeiten. Dabei spielt Technologie eine große Rolle, um transparent Informationen bereitzustellen. Softwaretools wie GlassFrog oder holaSpirit werden dabei unter anderem genutzt, um das Ziel und die Verantwortlichkeiten, aber auch den Fortschritt sowie die Entscheidungen der jeweiligen Circles miteinander zu kommunizieren und abzugleichen. Der gemeinsame Informationsstand in Self-Managed Organizations ist aufgrund der verteilten Natur, der Akteurinnen und Akteure, die in einer Organisation zusammenwirken, entscheidend. Bei Morning Star werden beispielsweise die Clous auf einem internen Server gespeichert, sodass Mitarbeiterinnen und Mitarbeiter transparent Informationen zu den Verantwortlichkeiten abrufen können. Wenn jemand sich in einer Rolle nicht bewährt, wird diese jemand anderem zugeteilt. Natürlich ist das Zuteilen von Rollen Arbeit an sich. In einer Holokratie gibt es auch dafür eine Rolle, die/ den sognannte/-n „Leadlink“, die/der die Aufgabe innehat, Circles miteinander zu verbinden. In noch flexibleren, loseren Formen des Selbstmanagements wie beispielsweise im Konzept der Podularity, werden Rollen ganz flexibel zugeteilt und die Art und Weise wie dies geschieht, wird der internen Organisation selbst überlassen. Zappos beispielsweise hat zweimal mehr „Leadlink“-Rollen als vorher Managerinnen und Manager angestellt waren. Dabei besteht der entscheidende Unterschied darin, dass die Führungsverantwortung jetzt zur Rolle gehört und nicht mehr zur individuellen Akteurin/ dem individuellen Akteur. Damit sind Autorität, Macht und Führungsverantwortung weiterhin vorhanden, jedoch hochkontextualisiert.

Insgesamt zeigt sich, dass Selbstorganisationsformen für große Organisationen und Unternehmen die Möglichkeit bieten, agile Strukturen in Teilen oder Gänze einzuführen. Die dafür zu nutzenden Konzeptionen sind neu, noch nicht in Gänze erprobt und deren Wirkungen bislang noch unbekannt. Jedoch liegt in jedem der Ansätze die Möglichkeit, bestehende traditionelle Strukturen zu hinterfragen, aufzubrechen und sowohl den Mitarbeiterinnen und Mitarbeitern als auch den Kundinnen und Kunden in ihren Fähigkeiten, Bedarfen und Anforderungen gerechter zu werden. Im Spannungsfeld zwischen Stabilität und Verlässlichkeit einerseits und Flexibilität und Beweglichkeit andererseits, gilt es nun also den richtigen Mix zu finden. Dabei sind Konzeptionen wie Holokratie, Podularity, 
Soziokratie sowie die demokratische Organisation, wichtige Konzeptionen, die die Gravitationszentren neuer moderner selbstorganisierter Unternehmens- und Organisationsstrukturen bilden. So unterschiedlich die einzelnen Ansätze sein mögen, es zeigt sich doch, dass sie alle versuchen, die individuellen Fähigkeiten von Organisationsmitgliedern mit den Rollen, den Strukturen und den Verantwortlichkeiten in der Organisation sowie mit den Organisationszielen besser und in größerer Flexibilität übereinzubringen und darauf Potentiale aufzuzeigen, wo flexibler Wandel stattfinden kann und soll. Das erfordert von den einzelnen Akteurinnen und Akteuren in hohem Maße Flexibilität, Wandlungsfähigkeit sowie Kompetenz und Selbstreflexion. Future Skills - das zeigt sich hier deutlich - sind in Bezug auf selbstorganisierte Unternehmen eine unerlässliche Voraussetzung. Darüber hinaus liegt in der Strukturierung von Organisationen als flexiblen Gebilden aber auch der Vorteil, dass sich Führungsrollen kontextuell über die Zeit hinweg verändern und wandeln können. Diese, fast spielerische Konzeption wird der Anforderung an Kompetenzen auf der einen Seite und dem, was Mitarbeiterinnen und Mitarbeiter mitbringen auf der anderen Seite, gerechter. Wichtig ist dabei, nicht den Fokus und die Transparenz zu verlieren, denn zwischen Circles, Pods und vielfältigen Aktionsformaten, gilt es stets den gemeinsamen Zweck im Auge zu behalten. Auch die Frage des Recruiting und der Gehälter ist in Self-Managed Organizations mit neuen und anderen Herausforderungen behaftet. In Situationen in denen Mitglieder ihre eigenen persönlichen Rollenportfolios bestimmen, ist es schwierig, klare Benchmarks oder marktgängige Gehälter zu bestimmen. Die Rollenentwicklung macht auch das Recruiting neuer Mitarbeiterinnen und Mitarbeiter kompliziert. So sind beispielsweise bei Zappos von Oktober bis Dezember 2015 von etwa 1.500 Mitarbeiterinnen und Mitarbeiter ca. 17.624 Rollen übernommen worden. Das entspricht rund elf Rollen pro Mitarbeiterin oder Mitarbeiter und 195 verschiedenen Rollen pro Tag. Die Vielfalt, Vielzahl und Vielgestaltigkeit dieser Rollen zu managen, transparent darzustellen, nachzuverfolgen und auch neue Mitarbeiterinnen und Mitarbeiter dabei einzubringen, $\mathrm{zu}$ „onboarden“ und überhaupt erst zu finden, macht eine komplett neue Vorgehensweise notwendig.

Traditionell werden Führungskräfte als diejenigen angesehen, die mit ihrer Vision Organisationen und Organisationsteile in die richtige Richtung steuern. Andererseits zeigt sich immer wieder, dass der Versuch, Organisationen mit einer Top-down-Konzeption zu verändern, nicht erfolgreich funktioniert. Im Harvard Business Review berichtet Rosabeth Moss Kanter in ihrem bekannten Artikel „Transforming Giants“ über die Frage „What enables a big business to be agile?“ (2008), dass der Erfolg von Change Prozessen in Unternehmen vor allem vom sogenannten "Guidance System“ oder den Navigationssystemen großer Organisationen abhänge. Während Mitarbeiterinnen und Mitarbeiter ursprünglich hauptsächlich 
nach Regeln und getroffenen Entscheidungen handelten, sind sie nun dazu angehalten, sich ganzheitlich einzubringen und an der Entwicklung eines geteilten Verständnisses und einer gemeinsamen Vision mitzuarbeiten. Die Bedeutsamkeit des Tuns nimmt einen weit größeren Stellenwert ein, genau wie die Identifizierung der Arbeitstätigkeit und das Alignment mit ihrer sie umgebenden Lebenswelt, ihren Partnerinnen und Partnern sowie der erweiterten Familie. Autorität und Führung in diesen neuen "Guidance Systems" wird aufrechterhalten und Aktivitäten koordiniert. Vor allem geht es aber um gemeinsam geteilte Werte und Standards und kohärente Organisationskulturen. Dieser Wandel zu neuen Guidance Systemen, so Kanter (2008), ist lange diskutiert und vorbereit worden und tritt nun mit erstaunlicher Geschwindigkeit ein. Ein Ausdruck dieser neuen Organisationsphilosophie findet sich im Gesamtbereich von Self-Managed Organizations. Schaut man sich so weitentwickelte Organisationformen an, wie beispielsweise Valve, wird die Selbstorganisation in vielen Unternehmensgeschichten deutlich. Beispielsweise in der Entscheidung, den Unternehmensmarkt über das Herstellen reiner PC-Spiele auch auf den Hardwarebereich auszuweiten. Bei Valve konzentrieren die über 400 Mitarbeiterinnen und Mitarbeiter ihre gesamte Zeit auf Projekte, die aus ihrer Sicht für ihre Kundinnen und Kunden wichtig sind. Sie arbeiten in Cabals zusammen, die sie selbst organisieren und reorganisieren Projekt für Projekt, indem sie ihre Stühle und Schreibtische zusammenstellen, manchmal mehrmals am Tag in neuer Form. (Natürlich ist es auch möglich, zu kundennah zu sein. Steve Jobs benannte dies einmal mit seiner berühmten Bemerkung, dass auch der Markt nicht immer wisse, was er wolle.)

In ihrem sehr guten Überblicksartikel im Harvard Business Review bemerken Ethan Bernstein, John Bunch, Nico Connor und Michael Lee (2016), dass breite Totschlagargumente für oder gegen Self-Managed Organizations oder Holokratie und andere neue Organisationsformen, in der Regel einen ganz wesentlichen Punkt außer Acht lassen: Die meisten Organisationen, gerade große Organisationen, sollten diese neuen Organisationsstrukturen und Arbeitstechniken in Teilen und nicht in Gänze implementieren. Sie bemerken:

"[W]e'd be surprised more than 20 percent of the Global 1000 looked 'teal' in 2030, to use Frederic Laloux's term for 'whole', evolutionary, self-managing organizations. But we'd also be surprised if more than twenty percent didn't significantly draw on some of the techniques within their corporate frameworks." (Bernstein, Bunch, Connor \& Lee 2016)

In den großen und kleinen Organisationen, sowohl den privaten als auch den öffentlichen, wird bereits viel mit Agilität und Selbstorganisation experimentiert. Procter $\&$ Gamble als Beispiel haben eine sehr komplexe Matrixstruktur implementiert, um 
ihre unterschiedlichen Produkte und Marken geografisch zu integrieren. Ergänzend dazu gibt es aber darüber hinaus ein sehr großes, ausgedehntes „Open Innovation Program“, in dem externen Team für Procter \& Gamble maßgeschneiderte Problemlösungen entwickeln. Google und $3 M$ sind ähnliche Beispiele: Für lange Zeit wurden die Mitarbeiterinnen und Mitarbeiter dazu ermutigt, einen bestimmten Prozentsatz als Anteil ihrer Arbeitszeit auf eigene Projekte (self directed work) zu verwenden. Die Frage in welcher Weise Selbstmanagement und Selbstorganisation in Unternehmen und anderen Organisationen eingeführt werden sollten und bis zu welchen Grad dies sinnvoll ist, kann anhand der Beantwortung dreier Fragen entschieden werden:

1. Was braucht es an Stabilität? Welche Teile der Organisation brauchen Stabilität?

2. Wo sind Anpassungen erforderlich und nötig?

3. Welche Organisationsformen sorgen für die richtige Balance?

Selbstmanagementprinzipien für gesamte Organisationen anzuwenden ist demnach dann sinnvoll, wenn der ideale Grad der Anpassungsfähigkeit sehr hoch ist. Wenn also die Organisation in einer sich schnell wandelnden Umwelt operiert, in der die Vorteile von schnellen flexiblen Anpassungen die Kosten dieser Anpassungsleistung übersteigen, außerdem Konsequenzen einer möglichen Fehlsteuerung und Falschanpassung nicht katastrophal wären und es drittens keinen hohen Bedarf an expliziter Kontrolle gäbe. Das ist auch der Grund dafür, dass vor allem Startups in diesem Bereich häufig zu den „early adopters“ zählen. Branchen wie Softwareentwicklungsorganisationen oder Spieleentwickler sind ebenfalls prototypisch für diese Kategorie, wie beispielsweise Valve entdeckte. Aber in Industrien, die ein hohes $\mathrm{Maß}$ an Verlässlichkeit charakterisiert - wie beispielsweise die Finanzbranche oder Verteidigungs- und militärische Organisationen - bleiben hierarchische Strukturen bestehen, obwohl es auch dort Nischenbereiche gäbe, in denen self-management fruchtbare Ansätze für eine zukunftsstarke Reorganisation darstellen würden.

\section{B 4.3 Fazit zum Thema Selbstorganisation als Grundprinzip}

Wir haben gezeigt, dass Selbstorganisation ein grundlegendes Prinzip von modernen Organisationsökosystemen ist. Dieses wirkt sowohl auf die Organisationsstrukturen (siehe agile Organisations- und Managementkonzepte), als auch auf die individuellen Akteurinnen und Akteure sowie auf den Bedarf an Skills und auch auf größere globale Strukturzusammenhänge, die sich wiederum gegen- 
seitig beeinflussen. Selbstorganisation geht zurück auf Prozesse im physikalisch naturwissenschaftlichen Bereich, indem Energiezufuhr zu nichtdeterministischen Phasenübergängen in Systemen führt. Überträgt man dies auf moderne Gesellschaften zeigt sich, dass mit Dirk Bäckers (2018) Analyse der Mediengesellschaft ein Informationsüberfluss die gleiche Wirkung auf soziale Systeme hat, die zu nichtdeterministischen Phasenübergängen, also Selbstorganisationsprozessen, führen. In einer Umgebung, in der Selbstorganisationsprozesse auf Märkten, in politischen Systemen und Organisationen sich durchsetzen und ermöglicht werden, werden sie zusammen mit der Fähigkeit, selbstorganisiert und eigenverantwortlich zu handeln, zu Voraussetzungen. Selbstorganisation schwingt sich damit zu einem Grundstrukturprinzip für die Entwicklung zukünftiger Fähigkeiten auf. 